\title{
Fatigue Failure Assessment of Metallic Specimens using the Acoustic Emission Technique.
}

\begin{abstract}
This paper presents a process to determine the relationship between acoustic emission (AE) signals and the strain signal pattern for estimating material fatigue life. In addition, it establishes the usefulness of $\mathrm{AE}$ in predicting the fatigue life of metallic components. This study was carried out to investigate the relationship between the strain and AE signals to ensure that $\mathrm{AE}$ can also be used as a tool to predict the fatigue life of metallic specimens. Two types of sensors, i. e., the AE piezoelectric transducer and the strain gauge, were attached to SAE 1045 steel specimens during the fatigue cyclic test when the specimens were under constant loading. Two types of signals were obtained at three different applied loads $(6.0 \mathrm{kN}, 6.4 \mathrm{kN}$ and $6.8 \mathrm{kN})$. Both signal types were then analyzed using a statistical model, and the results were used to correlate the fatigue life and AE signals. The fatigue life values were calculated using strain-life models. The correlation between the experimental and predicted values of fatigue life was then established. The results showed that the correlation between the r.m.s. AE values and the calculated fatigue life ranged from $74.0 \%$ to $98.5 \%$, while the correlation between the kurtosis $\mathrm{AE}$ values and the predicted values was found to be between $95.0 \%$ and $99.9 \%$. These correlation values showed that the AE technique can be used to predict the fatigue life of metallic specimens.
\end{abstract}

Keyword: Acoustic Emission 